\section{Remember to forget}

Memories do not always last—and their most fragile moments are when they are recalled. During recall, memories become transiently labile and can be forgotten. Two studies suggest how to harness this phenomenon to treat addiction.

Storing some forms of memory requires activation of signaling cascades and protein synthesis. Every time these memories are activated, they need to be reconsolidated. Injection of a protein-synthesis inhibitor in the brain of an animal while it is remembering a previously learned behavior does not affect its performance right then-but it impairs performance on the next trial. The inhibitor interferes with the reconsolidation process and erases the memory while it is active.

Drug addiction depends on associating environmental factors — such as drug-related paraphernalia — with the rewarding effects of drugs. Is it possible to interfere with the reconsolidation of these drug-related memories to wipe out addiction?

Jonathan Lee et al. (Neuron 47, 795-801) trained rats to self-administer cocaine in association with environmental cues and injected their brains with an antisense oligonucleotide against Egr1 (also known as Zif268), a gene important for the formation of drug-related memories. They found that the rats forgot their training and stopped drug-seeking behavior.

Courtney Miller and John Marshall (Neuron 47, 873-884) obtained similar results injecting a MEK kinase inhibitor in the brains of rats that had acquired a place preference in response to cocaine. The animals learned to associate the drug's rewarding effects with a specific location, choosing to spend more time in it. Interfering with reconsolidation of this memory abolished the place preference.

The two studies indicate that drug-related memories can become transiently labile when reactivated. Interfering with their reconsolidation might open a new therapeutic avenue for addiction. —JCL

\section{Skin is in}

Researchers have clashed for years over the cause of psoriasis: is it a disorder of the skin with immunological consequences, or an immune disorder that affects the skin? Unfortunately, there have been no good animal models in which to test these hypotheses. In the 15 September issue of Nature (437, 369-375), Rainer Zenz et al. wade into this debate with a new mouse model of psoriasis.

The researchers discovered that psoriatic human skin cells have decreased expression of JunB, a signaling molecule that can regulate cell proliferation; the gene that encodes JunB is localized to a psoriasissusceptibility region on chromosome 19 . They next created a mouse model by deleting a homolgous gene and one of its functional partners.

Analyses of the mice suggest that psoriasis arises as a disorder of the skin - for instance, blocking the immune response in the animals did not make the skin lesions disappear. - JB

\section{microRNA helps hepatitis C}

MicroRNAs are best known as negative regulators, binding to RNA and repressing translation or causing degradation. Catherine Jopling et al. turn that notion on its head, showing that a microRNA in the liver promotes the survival of hepatitis $\mathrm{C}$ virus, a major cause of chronic liver disease.

The researchers examined microRNA 122, a 21-22-nucleotide RNA expressed at blazing levels specifically in the liver. In the 2 September Science $(309,1577-1580)$, they report that cell lines with high levels of microRNA

Written by Allison Alcivar, Jasmine Bhatia, Eva Chmielnicki, Juan Carlos López and Charlotte Schubert
122 also support high levels of hepatitis $\mathrm{C}$ virus. They next found that the microRNA appears to bind specific sequences in the RNA of heptatis $\mathrm{C}$ virus. This binding, however, did not seem to affect mRNA translation or stability. Instead, the investigators propose, the microRNA may facilitate replication of the hepatitis $C$ virus RNA.

The results suggest that knocking down microRNA 122 in the liver might counteract infection with hepatitis $\mathrm{C}$ virus. - CS

\section{Exercise your neurons}

Getting to the gym is challenging for everyone, but as a recent study suggests, exercise is something that individuals with spinal muscular atrophy (SMA) cannot afford to avoid.

SMA is an inherited neuromuscular disease, affecting 1 in 10,000 live births. The cells of individuals with SMA have abnormally low levels of survival motor neuron (SMN) protein, which is required for motor neuron survival. Because of this defect, motor neurons die in the spinal cord of SMA patients.

Exercise is neuroprotective in many neurodegenerative and neuromuscular diseases. Likewise, in the 17 August Journal of Neuroscience (25, 7615-7622) Clément Grondard et al. found that

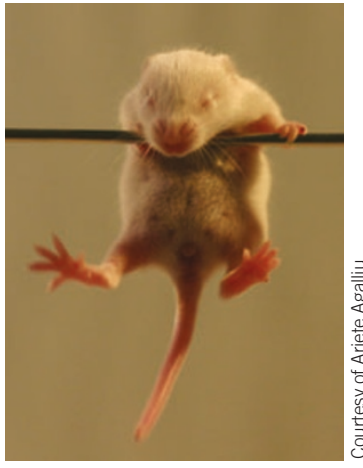

Mice with a neuromuscular disease have a stronger grip if they exercise regularly, because of changes in alternative splicing of a critical mRNA transcript. exercise improves the survival of SMA mice-and it occurs by an unusual mechanism.

The researchers found that trained SMA mice had more surviving motor neurons that projected to muscles, larger muscle fibers, and increased grip strength compared with untrained SMA mice. The trained mice also had alterations in splicing of a transcript encoding SMN. In SMA mice and individuals with the disease, the transcript lacks exon 7, which must be included in the SMN protein so that it can avoid degradation. In SMA mice, exercise upregulated the number of transcripts that included exon 7 and boosted the levels of SMN protein in motor neurons. How exercise induces changes in mRNA splicing remains to be explored. - EC

\section{Feel it in the bones}

Clock proteins, which operate in the circadian rhythm, mediate the formation and destruction of bones, according to a study in the 9 September Cell (122, 803-815). What's more, the hormone leptin-best known for regulating appetite - in turn controls the Clock proteins.

Previous work had suggested that bone remodeling — which involves cycles of bone formation and destruction-occurred in a circadian rhythm, with levels of osteocalcin, a bone protein, peaking at night. So Loning Fu et al. were not surprised to find that bone cells showed circadian fluctuations in the Clock proteins. The Clock proteins, they found, inhibited bone-cell proliferation through inhibition of cyclins. More surprisingly, the expression of the Clock genes was affected by leptin.

Leptin has recently been found to modulate bone formation, and the new work suggests that leptin does so by acting as both a positive and negative regulator. Leptin negatively regulates bone formation by upregulating expression of the Clock genes, and positively regulates bone formation by a separate pathway. The data suggest that the effect of leptin on the Clock genes might be specific to bone. The investigators speculate that bone remodeling might be a primary and ancestral function of leptin. - $A A$ 